Interfaces and Free Boundaries 14 (2013), 381-403

DOI 10.4171/IFB/307

\title{
On the interactions between a solid body and a compressible inviscid fluid
}

\author{
RAUL BORSCHE \\ Fachbereich Mathematik, Technische Universität Kaiserslautern, Germany \\ E-mail: borsche@mathematik.uni-kl.de \\ RiNALDO M. COLOMBO \\ Unità INDAM, Via Branze 38, 25123 Brescia, Italy \\ E-mail: rinaldo.colombo@unibs.it \\ Mauro Garavello \\ Dipartimento di Matematica e Applicazioni, Università di Milano - Bicocca, Via R. Cozzi 55, \\ 20125 Milano, Italy \\ E-mail:mauro.garavello@unimib.it
}

[Received 30 October 2012]

\begin{abstract}
This paper presents a model describing the interaction between a solid body and a compressible inviscid fluid in a pipe. The resulting system consists in a 1D hyperbolic balance law coupled with an ordinary differential equation and is proved to be well posed. Simple explicit solutions and numerical integrations show qualitative features of this model. In particular, we consider a ball falling in a vertical tube closed at the bottom. This system results in the ball bouncing on the shocks reflected between the ball and the bottom.
\end{abstract}

2010 Mathematics Subject Classification: Primary 35L65, 35M33; Secondary 34A12.

Keywords: Mixed PDE-ODE problems, balance laws, ordinary differential equations

\section{Introduction}

We consider a solid body moving in a tube filled with a non-viscous compressible fluid. Below, this situation is described through a hyperbolic 1D system of balance laws coupled with an ordinary differential equation. The former deals with the evolution of the fluid, modeled as one dimensional, while the latter captures the dynamics of the solid body, modeled as a point mass.

This mixed o.d.e-p.d.e. system, whose derivation is detailed in Section 2, fits in a suitable modification of the analytical framework in [3, 4]. Several physical situations enter the present theoretical setting: we recall, for instance, the flow in a sewer system with a manhole, see [3, § 3.2]; the flow of blood, see $[3, \S 3.4]$ or $[8,13]$; or a moving bottleneck in vehicular traffic, see $[4, \S 3.2]$ or [15].

From the physical point of view, the current literature offers several models for solid-fluid interactions. A point mass moving in an inviscid fluid is studied in [2]. The experimental works [1,7] deal with a solid ball falling in a stratified fluid. Remarkably, this rather different physical setting leads to a very similar trajectory for the falling body, see in particular Figure 4 below and [1, Figures 4, 5 and 6]. Indeed, a ball falling in a vertical tube filled with a compressible fluid causes a compression wave propagating downwards faster than the ball. This wave, focusing into a shock, bounces against the bottom of the tube, propagates upwards and hits the ball. As a result of this 
interaction, the acceleration of the ball changes discontinuously, resulting in stopping the fall of the ball, possibly also pushing it upwards. The repeated bounces of the shocks between the bottom of the tube and the ball allow to keep the ball suspended in the fluid for a while, see Section 4.2.

A relevant detail in the present model is its ability to allow for mass transfer between the two sides of the solid body. Indeed, in [3, 4], the conditions at the fluid-solid interfaces do not allow mass transfer. Here, the two interfaces on the sides of the solid are treated as free boundaries evolving according to the conservation of mass, to the balance of momentum and to the dissipation of energy. The main properties of the model so obtained are shown first through a few simple examples of explicit analytical solutions at the end of Section 2, then through numerical integrations of more complex situations in Section 4.

The analytical setting exploited below is developed in [3, 4]. The result obtained fits in the standard literature about $1 \mathrm{D}$ systems of conservation or balance laws, see for instance $[3,4,6,9,10]$. In particular, we select solutions that not only depend Lipschitz continuously from the data and various parameters, but also that are strongly stable, in both the $\mathbf{L}^{\infty}$ and $\mathbf{B V}$ sense, see 1 . in Theorem 3.6. Here, we consider in detail the case of the initial value problem, i.e., no fixed outer boundaries are present in the well posedness result below, see Theorem 3.6. Indeed, the analytical machinery necessary to pass to initial - boundary values problems is well established and this extension is of a merely technical nature, we refer to [5] for a sketch of it.

The next section is devoted to the derivation of the model. Section 3 deals with the analytical well posedness result, while Section 4 is devoted to the numerical integration of specific examples that show the main qualitative features of the model. All analytical details are deferred to the final Section 5.

\section{Model derivation}

Let $x$ denote the coordinate along the rectilinear tube, oriented as in Figure 1, and denote by $X=X(t)$ the position of the (center of the) solid body. The angle $\vartheta$ between the pipe and the horizontal plane is fixed throughout. The classical isentropic Euler equations, see for instance [11,
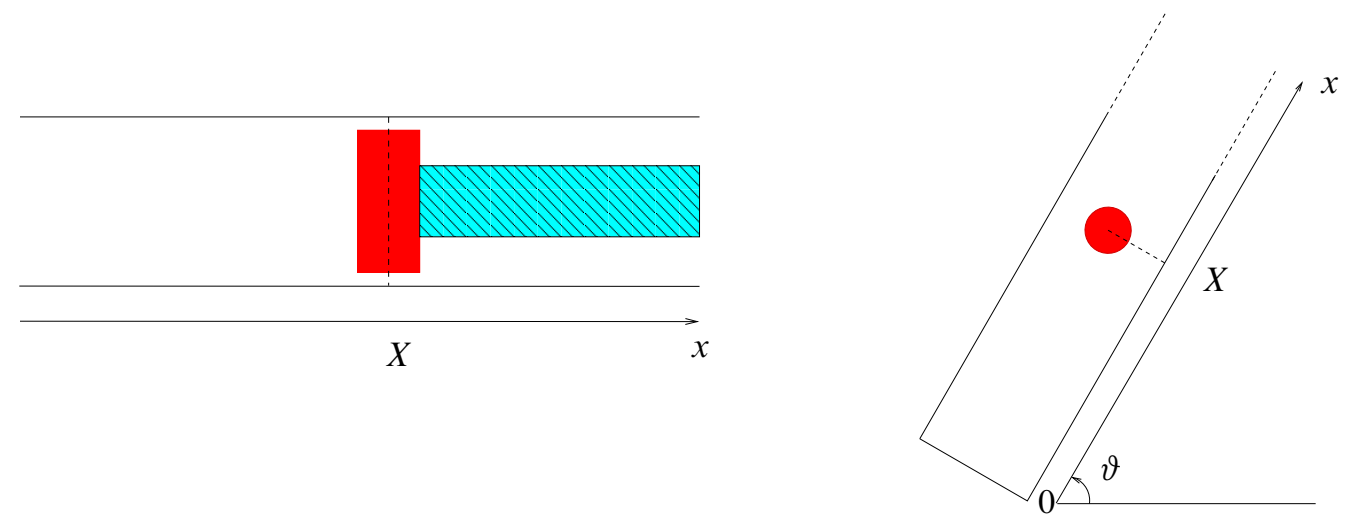

FIG. 1. Sketch of the situation described in (2.12). Left, a piston in a horizontal tube with no ends. Note the different sections available to the fluid on the two sides of the piston. Right, a falling ball in a tube closed at the bottom. 
Formula (7.1.9)],

$$
\left\{\begin{array}{l}
\partial_{t} \rho+\partial_{x} q=0 \\
\partial_{t} q+\partial_{x}\left(\frac{q^{2}}{\rho}+p(\rho)\right)=0
\end{array}\right.
$$

describe the fluid. Here, $\rho=\rho(t, x)$ is the fluid density, $q=q(t, x)$ is the linear momentum density and $p=p(\rho)$ is the pressure law characteristic of the fluid. Typical choices are

$$
\begin{array}{ll}
p(\rho)=p_{o} \cdot\left(\rho / \rho_{o}\right)^{\gamma} & \text { with } \quad \gamma>1, \quad p_{o}, \rho_{o}>0, \text { and } \\
p(\rho)=\sigma^{2} \rho & \text { with } \quad \sigma>0 .
\end{array}
$$

Recall the dynamic pressure, the total energy density and the energy flow related to (2.1), respectively given by:

$P(\rho, q)=\frac{q^{2}}{\rho}+p(\rho), \quad E(\rho, q)=\frac{q^{2}}{2 \rho}+\rho \int_{\rho_{*}}^{\rho} \frac{p(r)}{r^{2}} \mathrm{~d} r, \quad F(\rho, q)=\frac{q}{\rho}(E(\rho, q)+p(\rho))$,

where $\rho_{*}$ is a fixed reference density. When gravity is present, (2.1) is modified as follows

$$
\left\{\begin{array}{l}
\partial_{t} \rho+\partial_{x} q=0 \\
\partial_{t} q+\partial_{x}\left(\frac{q^{2}}{\rho}+p(\rho)\right)=-\rho g \sin \vartheta,
\end{array}\right.
$$

see for instance [10, Formula (3.1)]. Here, $g$ is gravity and the right hand side in the second equation in (2.5) describes the lack of linear momentum conservation due to $g$.

The solid of mass $m$ occupies the segment $[X(t)-\delta, X(t)+\delta]$, for a fixed $\delta>0$. Denote by $A=A(t, x)$ the section of the tube. For instance, in the case of the piston, see Figure 1, left, we have

$$
A(t, x)=A^{-} \chi_{]-\infty, X(t)-\delta[}(x)+A_{o} \chi_{[X(t)-\delta, X(t)+\delta]}(x)+A^{+} \chi_{] X(t)+\delta,+\infty[}(x) .
$$

To have a fully determined problem, we now seek further conditions to determine the function $X=X(t)$. We expect that, generically, 3 relations are necessary and sufficient to identify it. Indeed, in a subsonic setting, 2 equations select the waves issuing from each side of the solid towards the fluid, while a third constraint is an ordinary differential equation for $X$.

Introduce the total mass, total linear momentum and total energy:

$$
\begin{aligned}
m(t) & =\int_{\mathbb{R}} A(t, x) \rho(t, x) \mathrm{d} x+m \\
\mathcal{Q}(t) & =\int_{\mathbb{R}} A(t, x) q(t, x) \mathrm{d} x+m \dot{X}(t) \\
\mathcal{E}(t) & =\int_{\mathbb{R}} A(t, x)(E(\rho(t, x), q(t, x))+\rho(t, x) g x \sin \vartheta) \mathrm{d} x+m g X(t) \sin \vartheta+\frac{1}{2} m \dot{X}^{2}(t) .
\end{aligned}
$$

We prescribe the conservation of the total mass, the balance of the linear momentum with the gravitational impulse and the conservation of energy, namely:

$$
\dot{m}(t)=0, \quad \dot{Q}(t)=-m g \sin \vartheta \quad \text { and } \quad \dot{\varepsilon}(t)=0 .
$$


Formally, setting $\rho(X+\delta \pm)=\lim _{\varepsilon \rightarrow 0 \pm} \rho(X+\delta+\varepsilon)$, we obtain

$$
\begin{aligned}
& \dot{X}\left(A^{+} \rho(X+\delta+)-A_{o} \rho(X+\delta-)+A_{o} \rho(X-\delta+)-A^{-} \rho(X-\delta-)\right) \\
& =A^{+} q^{+}(X+\delta+)-A_{o} q(X+\delta-)+A_{o} q(X-\delta+)-A^{-} q(X-\delta-) \text {; } \\
& \dot{X}\left(A^{+} q(X+\delta+)-A_{o} q(X+\delta-)+A_{o} q(X-\delta+)-A^{-} q(X-\delta-)\right) \\
& =A^{+} P^{+}(X+\delta+)-A_{o} P(X+\delta-)+A_{o} P(X-\delta+)-A^{-} P(X-\delta-)+m \ddot{X} \\
& +m g \sin \vartheta \\
& \dot{X}\left(A^{+} E(X+\delta+)-A_{o} E(X+\delta-)+A_{o} E(X-\delta+)-A^{-} E(X-\delta-)\right) \\
& =A^{+} F^{+}(X+\delta+)-A_{o} F(X+\delta-)+A_{o} F(X-\delta+)-A^{-} F(X-\delta-) \\
& +m(\ddot{X}+g \sin \vartheta) \dot{X} \text {. }
\end{aligned}
$$

We now assume that $\delta$ is so small that waves propagate between $X \pm \delta$ to $X \mp \delta$ instantaneously, so that for a.e. $t \in \mathbb{R}^{+},(\rho, q)(t, X(t)+\delta-)=(\rho, q)(t, X(t)-\delta+)$ and hence we get the conditions

$$
\begin{aligned}
\dot{X} \Delta(A \rho)-\Delta(A q) & =0, \\
\dot{X} \Delta(A q)-\Delta(A P) & =m(\ddot{X}+g \sin \vartheta), \\
\dot{X} \Delta(A E)-\Delta(A F) & =m(\ddot{X}+g \sin \vartheta) \dot{X}
\end{aligned}
$$

Above, we used the following notation: for any quantity $G=G(\rho, q)$ we set

$$
\Delta G(t)=G(\rho(t, X(t)+), q(t, X(t)+))-G(\rho(t, X(t)-), q(t, X(t)-)) .
$$

Moreover, in the limit $\delta \rightarrow 0$ the section of the tube is

$$
A(t, x)=A^{-} \chi_{]-\infty, X(t)[}(x)+A^{+} \chi_{] X(t),+\infty[}(x) .
$$

We are thus led to study the problem consisting of (2.5), (2.8), (2.9) and (2.10), which we rewrite thanks to (2.4) as

$$
\left\{\begin{array}{l}
\partial_{t} \rho+\partial_{x} q=0 \\
\partial_{t} q+\partial_{x} P=-\rho g \sin \vartheta \\
\ddot{X}=-g \sin \vartheta-\frac{1}{m}(\Delta(A P)-\dot{X} \Delta(A q)) \\
\Delta(A q)-\dot{X} \Delta(A \rho)=0 \\
\Delta(A F)-\dot{X} \Delta(A E)=\dot{X}(\Delta(A P)-\dot{X} \Delta(A q)) .
\end{array}\right.
$$

Note that the ordinary differential equation on the third line of (2.12) is coherent with the Archimedean law. Indeed, in the hydrostatic case $q \equiv 0$ and $\dot{X} \equiv 0$, the weight $m g \sin \vartheta$ of the solid body is balanced by the force $\Delta(A p)$.

When the effects of friction can not be neglected, the $p$-system (2.5) now reads

$$
\left\{\begin{array}{l}
\partial_{t} \rho+\partial_{x} q=0 \\
\partial_{t} q+\partial_{x} P=-\rho g \sin \vartheta-v_{F}(\rho, q)
\end{array}\right.
$$


where the term $v_{F}(\rho, q)$ describes the friction between the fluid and the pipe's walls. The local balance of momentum (2.9) and energy (2.10) at $X$ become

$$
\begin{aligned}
\dot{X} \Delta(A q)-\Delta(A P) & =m(\ddot{X}+g \sin \vartheta)+m v_{S}(\dot{X}) \\
\dot{X} \Delta(A E)-\Delta(A F) & =m(\ddot{X}+g \sin \vartheta) \dot{X}+m \dot{X} v_{S}(\dot{X})+v_{I}(q / \rho, \dot{X})
\end{aligned}
$$

where $v_{S}$ quantifies the friction between the solid and the pipe's walls; $v_{I}(q / \rho, \dot{X})$ accounts for the friction due to the solid-fluid interaction. Possible standard choices are, for instance,

$$
v_{F}(\rho, q)=\tilde{v}_{F} \frac{q|q|}{\rho}, \quad v_{S}(\dot{X})=\tilde{v}_{S} \dot{X} \quad \text { and } \quad v_{I}(q / \rho, \dot{X})=\tilde{v}_{I}\left(\dot{X}-\left\langle\frac{q}{\rho}\right\rangle\right)^{2}
$$

for suitable positive $\tilde{v}_{F}, \tilde{v}_{S}$ and $\tilde{v}_{I}$. Here, we denote $\left\langle\frac{q}{\rho}\right\rangle=\frac{1}{2}\left(\frac{q(t, X(t)+)}{\rho(t, X(t)+)}+\frac{q(t, X(t)-)}{\rho(t, X(t)-)}\right)$. We thus pass to the system

$$
\left\{\begin{array}{l}
\partial_{t} \rho+\partial_{x} q=0 \\
\partial_{t} q+\partial_{x} P=-\rho g \sin \vartheta-v_{F}(\rho, q) \\
\ddot{X}=-g \sin \vartheta-\frac{1}{m}(\Delta(A P)-\dot{X} \Delta(A q))-v_{S}(\dot{X}) \\
\Delta(A q)-\dot{X} \Delta(A \rho)=0 \\
\Delta(A F)-\dot{X} \Delta(A E)=\dot{X}(\Delta(A P)-\dot{X} \Delta(A q))-v_{I}(q / \rho, \dot{X}) .
\end{array}\right.
$$

We prove in Lemma 3.3 the following estimates on the variations of total mass, momentum and energy along the solutions to (2.14), also in the case of weak entropy solutions:

$$
\begin{aligned}
& \dot{m}(t)=0 \\
& \dot{Q}(t)=-\int_{\mathbb{R}} A(t, x) v_{F}(\rho, q) \mathrm{d} x-m v_{S}(\dot{X})-m g \sin \vartheta \\
& \dot{\varepsilon}(t) \leqslant-\int_{\mathbb{R}} A(t, x) \frac{q(t, x)}{\rho(t, x)} v_{F}(\rho, q) \mathrm{d} x-m v_{S}(\dot{X}) \dot{X}-v_{I}(q / \rho, \dot{X})
\end{aligned}
$$

As expected, the total mass in conserved. The usual conditions

$$
q v_{F}(\rho, q) \geqslant 0, \quad \dot{X} v_{S}(\dot{X}) \geqslant 0 \quad \text { and } \quad v_{I}(q / \rho, \dot{X}) \geqslant 0
$$

on the friction terms ensure that the total energy decreases. Besides, (2.16) also implies, for instance, that if $q(t, x)>0$ for all $x$ and $\dot{X}(t)>0$, then $\dot{Q}(t)<0$.

Remark that in (2.15) the variation $\dot{Q}$ of the linear momentum is not affected by $v_{I}(q / \rho, \dot{X})$. In fact, this term describes the effect of the interaction between the solid and the fluid, hence of internal forces.

A further property of (2.14) is that it is Galileian invariant, as soon as $v_{F} \equiv 0, v_{S} \equiv 0$ and $v_{I}$ is a function of the difference $\dot{X}-\langle q / \rho\rangle$, as for instance in (2.13), see Lemma 3.4.

The next examples display explicit solutions to (2.14), thus showing features of the present model.

EXAMPLE 2.1 With the pressure law (2.3), if $A^{+}=A^{-}$in (2.11), $v_{F}(\rho, 0)=0$ and $v_{I}=0$, then a simple solution to (2.14) is

$$
\begin{aligned}
& \rho(t, x)=\bar{\rho} \exp \left(-\frac{g \sin \vartheta}{\sigma^{2}}(x-\bar{X})\right) \\
& q(t, x)=0
\end{aligned} \text { and } X=X(t) \text { solves }\left\{\begin{array}{l}
\ddot{X}+v_{S}(\dot{X})=-g \sin \vartheta \\
X(0)=\bar{X} \\
\dot{X}(0)=\bar{V}
\end{array}\right.
$$


where $\bar{\rho}>0$ and $\bar{X}, \bar{V} \in \mathbb{R}$. This solution describes a fluid in hydrostatic equilibrium with a solid body falling through it, due to gravity and subject to the friction against the pipe walls. Note that the fluid and the solid body are completely independent and moving with respect to each other. This somewhat artificial solution does not meet the stability requirements (3.5)-(3.6) necessary for Theorem 3.6, see Lemma 5.3.

EXAMPLE 2.2 Consider a piston in a pipe filled with fluid satisfying the pressure law (2.3), if $v_{F}=v_{S}=0$ and $v_{I}(q / \rho, \dot{X})=\bar{v}_{I}(\dot{X}-q / \rho)$ with $\bar{v}_{I}(0)=0$, direct computations show that

$$
\begin{aligned}
& \rho(t, x)=\left\{\begin{array}{ll}
\rho^{-} \exp \left(-\frac{g \sin \vartheta}{\sigma^{2}}\left(x-X_{o}\right)\right) & x<X_{o}, \\
\rho^{+} \exp \left(-\frac{g \sin \vartheta}{\sigma^{2}}\left(x-X_{o}\right)\right) & x>X_{o},
\end{array} \quad \text { and } \quad \begin{array}{c}
X(t)=X_{o} \\
q(t, x)=0,
\end{array}\right. \\
& \dot{X}(t)=0
\end{aligned}
$$

solves (2.14) if and only if the Archimedean force balances the weight of the solid, i.e.,

$$
m g \sin \vartheta=\sigma^{2}\left(A^{-} \rho^{-}-A^{+} \rho^{+}\right) .
$$

In this static solution, the piston is in equilibrium in the middle of the fluid. Other (non static) traveling wave solutions can be obtained applying Galileian transformations, see Lemma 3.4. All these solutions are stable in the sense that the conditions (3.5)-(3.6) are satisfied, as proved in Lemma 5.4.

EXAMPLE 2.3 With the pressure law (2.3), if $v_{F}=v_{S}=v_{I}=0$, a stationary solution to (2.14) with different sections $A^{ \pm}$and with mass transfer between the two sides of the solid is as follows:

$$
\rho(t, x)=\left\{\begin{array}{ll}
\rho^{-} & x<X_{o} \\
\rho^{+} & x>X_{o}
\end{array} \quad q(t, x)=\left\{\begin{array}{ll}
q^{-} & x<X_{o} \\
q^{+} & x>X_{o}
\end{array} \quad X(t)=X_{o},\right.\right.
$$

where $X_{o} \in \mathbb{R}, A^{-}>0, \rho^{-}>0, q^{-} \in \mathbb{R}$ and

$$
\begin{aligned}
A^{+} & =\xi \exp \left(\frac{1-\xi^{2}}{2 \xi}\right) \\
\rho^{+} & =\frac{A^{-}}{A^{+}} \xi \rho^{-} \quad \text { where } \quad \xi=\left(\frac{q^{-}}{\sigma \rho^{-}}\right)^{2} . \\
q^{+} & =\frac{A^{-}}{A^{+}} q^{-}
\end{aligned}
$$

This solution does not satisfy the subsonic condition (3.11). Elementary computations show that if the left state $\left(\rho^{-}, q^{-}\right)$is subsonic, then the right state $\left(\rho^{+}, q^{+}\right)$is supersonic.

In all the examples above, the choice (2.3) of the pressure law is necessary only to allow the explicit writing of the solutions to the $p$-system (2.1). Indeed, Lemma 5.2 ensures that analogous solutions may exist under more general assumption on the pressure law.

\section{Analytical results}

Throughout, $\mathbb{R}^{+}=\left[0,+\infty\left[\right.\right.$ and $\left.\mathbb{R}^{+}=\right] 0,+\infty[$. To simplify the notation, we identify the state $(\rho, q)$ with $u$, so that for instance $u^{ \pm}=\left(\rho^{ \pm}, q^{ \pm}\right)$. The ball in $\mathbb{R}^{2}$ centered at $u$ with radius $\delta>0$ is denoted by $B(u, \delta)$. For the basic results in the theory of hyperbolic conservation laws we refer to [11], while for those concerning Caratheodory differential equations, see [14].

The pressure law $p=p(\rho)$ is assumed to satisfy the following standard assumption: 
(p) $p \in \mathbf{C}^{2}\left(\mathbb{R}^{+} ; \mathbb{R}^{+}\right)$is such that $p^{\prime}(\rho)>0$ and $p^{\prime \prime}(\rho) \geqslant 0$ for all $\rho>0$.

Both pressure laws (2.2) and (2.3) satisfy (p).

As a first step, we formalize what we mean by solution to (2.14).

Definition 3.1 Let $T>0$ be fixed, $u_{o} \in \mathbf{B V}\left(\mathbb{R} ; \mathbb{R}^{+} \times \mathbb{R}\right), X_{o} \in \mathbb{R}$ and $V_{o} \in \mathbb{R}$. The map $(u, X)$ is a solution to (2.14) with initial condition

$$
\left\{\begin{aligned}
u(0, x) & =u_{o}(x) \\
X(0) & =X_{o} \\
\dot{X}(0) & =V_{o}
\end{aligned}\right.
$$

on the time interval $[0, T]$ if

1. $u \in \mathbf{C}^{\mathbf{0}}\left([0, T] ; \mathbf{B V}\left(\mathbb{R} ; \mathbb{R}^{+} \times \mathbb{R}\right)\right), X \in \mathbf{W}^{\mathbf{1}, \infty}([0, T] ; \mathbb{R})$ and $\dot{X} \in \mathbf{W}^{\mathbf{1}, \mathbf{1}}([0, T] ; \mathbb{R})$.

2. $u$ is a weak entropy solution to

$$
\left\{\begin{array}{l}
\partial_{t} \rho+\partial_{x} q=0 \\
\partial_{t} q+\partial_{x} P(\rho, q)=-\rho g \sin \vartheta-v_{F}(\rho, q)
\end{array}\right.
$$

for $(t, x) \in[0, T] \times]-\infty, X(t)[$ and for $(t, x) \in[0, T] \times] X(t),+\infty[$.

3. $X$ solves

$$
\ddot{X}=-g \sin \vartheta-\frac{1}{m}(\Delta(A P)-\dot{X} \Delta(A q))-v_{S}(\dot{X})
$$

in Caratheodory sense for $t \in[0, T]$.

4. For a.e. $t \in[0, T]$, the boundary conditions

$$
\left\{\begin{array}{l}
\Delta(A q)=\dot{X} \Delta(A \rho) \\
\Delta(A F)-\dot{X} \Delta(A E)=\dot{X}(\Delta(A P)-\dot{X} \Delta(A q))-v_{I}(q / \rho, \dot{X})
\end{array}\right.
$$

are satisfied in the sense of the traces at $X(t)^{ \pm}$.

5. The initial condition is satisfied in the strong sense: $u(0, x)=u_{o}(x)$ for a.e. $x \in \mathbb{R}, X(0)=X_{o}$ and $\dot{X}(0)=V_{o}$.

Here, at 1 ., the continuity of $u$ is understood in the strong $\mathbf{L}^{1}$ topology. At 2., we mean that for all $\varphi \in \mathbf{C}_{\mathbf{c}}^{\mathbf{1}}(] 0, T[\times \mathbb{R} ; \mathbb{R})$ such that

$$
\operatorname{spt} \varphi \cap\{(t, x) \in] 0, T\left[\times \mathbb{R}^{+}: x=X(t)\right\}=\emptyset,
$$

the following relation holds:

$$
\int_{0}^{T} \int_{\mathbb{R}}\left(\left[\begin{array}{l}
\rho \\
q
\end{array}\right] \partial_{t} \varphi+\left[\begin{array}{c}
q \\
P(\rho, q)
\end{array}\right] \partial_{x} \varphi+\left[\begin{array}{c}
0 \\
-\rho g \sin \vartheta-v_{F}(\rho, q)
\end{array}\right] \varphi\right) \mathrm{d} x \mathrm{~d} t=0
$$

and for all $\varphi \in \mathbf{C}_{\mathbf{c}}^{\mathbf{1}}(] 0, T\left[\times \mathbb{R} ; \mathbb{R}^{+}\right)$satisfying (3.2), for any convex entropy - entropy flux pair $(\eta, \chi)$, see [6, Definition 4.4], the inequality

$$
\int_{0}^{T} \int_{\mathbb{R}}\left(\eta(\rho, q) \partial_{t} \varphi+\chi(\rho, q) \partial_{x} \varphi+D \eta(\rho, q)\left[\begin{array}{c}
0 \\
-\rho g \sin \vartheta-v_{F}(\rho, q)
\end{array}\right] \varphi\right) \mathrm{d} x \mathrm{~d} t \geqslant 0
$$

holds. Concerning 4., remark that the traces of $u$ at $X(t) \pm$ exist, thanks to the requirement $u \in \mathbf{B V}$.

The present framework, based on (2.12) and on Definition 3.1, comprises [4, Section 3.1], as proved by the following lemma. 
Lemma 3.2 Let $p$ satisfy (p), fix positive $g, m, \tilde{v}_{F}, \tilde{v}_{S}$ and $\vartheta \in[0, \pi / 2]$ and set $A^{+}=A^{-}$ in (2.11). Let $(u, X)$ solve [4, Formula (1.2)], i.e.,

$$
\left\{\begin{array}{l}
\partial_{t} \rho+\partial_{x} q=0, \\
\partial_{t} q+\partial_{x} P=-\rho g \sin \vartheta-\tilde{v}_{F} \frac{q|q|}{\rho^{2}}, \\
\ddot{X}=-g \sin \vartheta-\frac{A^{+}}{m} \Delta p-\tilde{v}_{S} \dot{X}, \\
\dot{X}=\frac{q(t, X(t)-)}{\rho(t, X(t)-)} \\
\dot{X}=\frac{q(t, X(t)+)}{\rho(t, X(t)+)}
\end{array}\right.
$$

in the sense of [4, Definition 2.1], then $(u, X)$ solves (2.14), in the case $v_{I}=0, v_{S}(\dot{X})=\tilde{v}_{S} \dot{X}$ and $v_{F}(\rho, q)=\tilde{v}_{F} q|q| / \rho^{2}$, in the sense of Definition 3.1.

The proof is immediate and, hence, omitted. Remark that the present setting allows the transfer of mass between the two sides of the solid body, which is impossible in (3.3). Therefore, the present construction is able to comprise, for instance, the situation in Figure 3, right.

We now pass to the rigorous statement of the conservation of mass, energy and the balance of momentum.

Lemma 3.3 Let $p$ satisfy (p), $p(0)=0$ and $A$ be as in (2.11) for fixed $A^{ \pm}>0$. Fix positive $g, m$ and $\vartheta \in[0, \pi / 2]$. Let $(u, X)$ solve (2.14)-(3.1) in the sense of Definition 3.1. Assume

$$
\begin{array}{ll}
\rho(t, x)>0, & \forall(t, x) \in \mathbb{R}^{+} \times \mathbb{R}, \\
\lim _{x \rightarrow \pm \infty} x \rho(t, x)=\lim _{x \rightarrow \pm \infty} \frac{q(t, x)}{\rho(t, x)}=0, & \forall t \geqslant 0, \\
x \rightarrow x \rho(t, x), x \rightarrow q(t, x), x \rightarrow \rho(t, x) \ln (\rho(t, x)) \in \mathbf{L}^{\mathbf{1}}(\mathbb{R}), & \forall t \geqslant 0 .
\end{array}
$$

Then, with reference to the quantities defined in (2.7), the inequalities (2.15) hold in distributional sense.

The proof is deferred to Section 5 .

The next lemma is devoted to the Galileian invariance of (2.12).

LEMmA 3.4 Let $v_{F}=v_{S}=0$ and $v_{I}(q / \rho, \dot{X})=\bar{v}_{I}(\dot{X}-q / \rho)$, with $\bar{v}_{I} \in \mathbf{C}_{\mathrm{loc}}^{0,1}(\mathbb{R} ; \mathbb{R})$. Let $(\rho, q, X)$ solve (2.14). Then, for any $\hat{X}, \hat{V}$ in $\mathbb{R}$, also the function $(\check{\rho}, \check{q}, \check{X})$ given by

$$
\begin{aligned}
& \check{\rho}(t, x)=\rho(t, x+\hat{X}+\hat{V} t) \\
& \check{q}(t, x)=q(t, x+\hat{X}+\hat{V} t)-\rho(t, x+\hat{X}+\hat{V} t) \hat{V} \quad \text { and } \quad \check{X}=X(t)-\hat{X}-\hat{V} t
\end{aligned}
$$

solves (2.14) in the sense of Definition 3.1.

The proof is straightforward and, hence, omitted.

Introduce the function $\Phi:\left(\mathbb{R}^{+} \times \mathbb{R}\right)^{2} \times \mathbb{R} \rightarrow \mathbb{R}^{2}$ whose components are defined by

$$
\begin{aligned}
& \Phi_{1}\left(u^{-}, u^{+}, V\right)=\Delta(A q)-\Delta(A \rho) V, \\
& \Phi_{2}\left(u^{-}, u^{+}, V\right)=\Delta(A F)-V \Delta(A E)-V(\Delta(A P)-V \Delta(A q))+v_{I}(q / \rho, V)
\end{aligned}
$$


with $P, E, F$ as in (2.4), so that the last two conditions in (2.14) can be concisely rewritten as

$$
\Phi(u(t, X(t)-), u(t, X(t)+), \dot{X}(t))=0 .
$$

Proposition 3.5 Let (p) hold and define $\Phi$ as in (3.4). Fix the positive parameters $m, g$ and $\vartheta$. Choose $v_{I} \in \mathbf{C}^{\mathbf{1}}(\mathbb{R} \times \mathbb{R} ; \mathbb{R})$. Assume there exist states $\bar{u}^{ \pm} \in \stackrel{\mathbb{R}}{ }^{+} \times \mathbb{R}$, a position $\bar{X} \in \mathbb{R}^{+}$and a speed $\bar{V} \in \mathbb{R}$ such that $\Phi\left(\bar{u}^{-}, \bar{u}^{+}, \bar{V}\right)=0$. Assume moreover that

$$
\begin{aligned}
& \operatorname{det}\left[D_{u^{-}} \Phi\left(\bar{u}^{-}, \bar{u}^{+}, \bar{V}\right) r_{1}\left(u^{-}\right) \quad D_{u^{+}} \Phi\left(\bar{u}^{-}, \bar{u}^{+}, \bar{V}\right) r_{2}\left(u^{+}\right)\right] \neq 0, \\
& \left(\left[D_{u^{-}} \Phi\left(\bar{u}^{-}, \bar{u}^{+}, \bar{V}\right) r_{1}\left(\bar{u}^{-}\right) \quad D_{u^{+}} \Phi\left(\bar{u}^{-}, \bar{u}^{+}, \bar{V}\right) r_{2}\left(\bar{u}^{+}\right)\right]^{-1} D_{V} \Phi\left(\bar{u}^{-}, \bar{u}^{+}, \bar{V}\right)\right)_{1,2} \neq 0 .
\end{aligned}
$$

Then, there exists a positive $\delta$ such that for all $u^{ \pm} \in B\left(\bar{u}^{ \pm}, \delta\right)$ and $\left.V \in\right] \bar{V}-\delta, \bar{V}+\delta[$ the two sets of conditions

$$
\Phi\left(u^{-}, u^{+}, V\right)=0 \quad \text { and } \quad\left\{\begin{array}{l}
b^{-}\left(u^{-}, u^{+}\right)=V \\
b^{+}\left(u^{-}, u^{+}\right)=V
\end{array}\right.
$$

are equivalent, for suitable smooth functions $b^{ \pm}$that also satisfy

$$
\nabla_{u^{-}} b^{-}\left(u^{-}, u^{+}\right) r_{1}\left(u^{-}\right) \neq 0, \quad \text { and } \quad \nabla_{u^{+}} b^{+}\left(u^{-}, u^{+}\right) r_{2}\left(u^{+}\right) \neq 0 .
$$

The proof is deferred to Section 5 .

The well posedness of (2.14) now immediately follows through [4, Theorem 2.7]. To state it, for fixed $\bar{u} \in \mathbb{R}^{+} \times \mathbb{R}, \bar{V} \in \mathbb{R}$, and $\delta>0$, we introduce the set

$$
u(\bar{u}, \bar{X}, \bar{V}, \delta)=\left\{(u, X, V):\left\{\begin{array}{l}
u \in \bar{u}+\mathbf{L}^{\mathbf{1}}\left(\mathbb{R}^{+} ; \mathbb{R}^{\circ} \times \mathbb{R}^{+}\right) \\
X \in \mathbb{R} \\
V \in \mathbb{R}
\end{array} \text { and }\left\{\begin{array}{c}
\mathrm{TV}(u)<\delta \\
|V-\bar{V}|<\delta
\end{array}\right\}\right.\right.
$$

where we define $\operatorname{TV}(u)=\operatorname{TV}(\rho)+\operatorname{TV}(q)$.

THEOREM 3.6 Let (p) hold and assume that $v_{F} \in \mathbf{C}^{\mathbf{0}, 1}\left(\stackrel{R}{ }^{+} \times \mathbb{R} ; \mathbb{R}\right), v_{S} \in \mathbf{C}_{\text {loc }}^{0,1}(\mathbb{R} ; \mathbb{R})$ and $v_{I} \in$ $\mathbf{C}^{\mathbf{1}}(\mathbb{R} \times \mathbb{R} ; \mathbb{R})$. Define $\Phi$ as in (3.4). Fix the parameters $m, g \in \mathbb{R}^{+}$and $\vartheta \in[0, \pi / 2]$. Assume there exist states $\bar{u}^{ \pm} \in \mathbb{R}^{+} \times \mathbb{R}$, a position $\bar{X} \in \mathbb{R}^{+}$and a speed $\bar{V} \in \mathbb{R}$ such that $\Phi\left(\bar{u}^{-}, \bar{u}^{+}, \bar{V}\right)=0$. Assume moreover that (3.5) and (3.6) hold. Then, system (2.14) can be rewritten

$$
\begin{cases}\partial_{t} u^{-}+\partial_{x} f^{-}\left(u^{-}\right)=\varphi^{-}\left(u^{-}\right) & x<\gamma^{-}(t), \\ \partial_{t} u^{+}+\partial_{x} f^{+}\left(u^{+}\right)=\varphi^{+}\left(u^{+}\right) & x>\gamma^{+}(t), \\ b^{-}\left(u^{-}\left(t, \gamma^{-}(t)-\right), u^{+}\left(t, \gamma^{+}(t)+\right)\right)=w(t), & \\ b^{+}\left(u^{-}\left(t, \gamma^{-}(t)-\right), u^{+}\left(t, \gamma^{+}(t)+\right)\right)=w(t), & \\ \dot{w}=\mathcal{F}\left(t, u^{-}\left(t, \gamma^{-}(t)-\right), u^{+}\left(t, \gamma^{+}(t)+\right), w(t)\right), & \\ \dot{\gamma}^{-}(t)=w(t), & \\ \dot{\gamma}^{+}(t)=w(t), & \end{cases}
$$


where

$$
\begin{array}{rlrl}
u^{ \pm} & =\left[\begin{array}{l}
\rho^{ \pm} \\
q^{ \pm}
\end{array}\right] & f^{ \pm}(u) & =\left[\begin{array}{c}
q \\
P(\rho, q)
\end{array}\right] \\
\gamma & =X & \mathcal{F}\left(t, u^{-}, u^{+}, w\right) & =-g \sin \vartheta-\frac{1}{m}(\Delta(A P)-\dot{X} \Delta(A q))-v_{S}(\dot{X}) \\
w & =\dot{X} & \varphi^{ \pm}(u) & =\left[\begin{array}{cc}
0 \\
-g \sin \vartheta \rho-v_{F}(\rho, q)
\end{array}\right]
\end{array}
$$

and with $b^{ \pm}$given by Proposition 3.5. Hence, under the subsonic condition

$$
|\bar{V}|<\sqrt{p^{\prime}\left(\bar{\rho}^{-}\right)} \text {and }|\bar{V}|<\sqrt{p^{\prime}\left(\bar{\rho}^{+}\right)},
$$

there exist positive $\delta, \Delta, L, T_{\delta}$, domains $Ð$ and a map $S:\left[0, T_{\delta}\right] \times \nsupseteq \mapsto \Phi$ such that the following holds:

1. $U(\bar{u}, \bar{V}, \delta) \subseteq \Phi \subseteq U(\bar{u}, \bar{V}, \Delta)$;

2. $S$ is a local semigroup, i.e., $S_{0}=\mathbf{I d}$ and $S_{t_{1}} \circ S_{t_{2}}=S_{t_{1}+t_{2}}$ for all $t_{1}, t_{2} \in\left[0, T_{\delta}\right]$ with $t_{1}+t_{2} \in\left[0, T_{\delta}\right]$

3. $S$ is Lipschitz, i.e., for all $\left(u_{1}, X_{1}, V_{1}\right),\left(u_{2}, X_{2}, V_{2}\right) \in \Phi$ and for all $t_{1}, t_{2} \in\left[0, T_{\delta}\right]$,

$$
\left\|S_{t_{2}}\left(u_{2}, X_{2}, V_{2}\right)-S_{t_{1}}\left(u_{1}, X_{1}, V_{1}\right)\right\| \leqslant L \cdot\left(\left\|u_{2}-u_{1}\right\|_{\mathbf{L}^{1}}+\left|X_{2}-X_{1}\right|+\left|V_{2}-V_{1}\right|+\left|t_{2}-t_{1}\right|\right) ;
$$

4. for all $\left(u_{o}, X_{o}, V_{o}\right) \in \Phi$, the orbit $t \rightarrow S_{t}\left(u_{o}, X_{o}, V_{o}\right)$ is a solution to (2.14) in the sense of Definition 3.1, for all $t \in\left[0, T_{\delta}\right]$.

Thanks to Proposition 3.5, the proof is an immediate application of [4, Theorem 2.7] and is hence omitted.

The next lemma ensures that the assumptions of Theorem 3.6 are non void.

Lemma 3.7 Consider the pressure law (2.3) with $\sigma=5$ and assume that $A^{-}=A^{+}$and $v_{I}=0$. Define

$$
\begin{array}{ll}
\bar{\rho}^{-}=2 & \bar{\rho}^{+}=9 / 5 \\
\bar{q}^{-}=1 & \bar{q}^{+}=\frac{171+90 \sqrt{38 \ln (10 / 9)}}{190}
\end{array}
$$

Then, the subsonic states $\bar{u}^{-}, \bar{u}^{+}$and the velocity $\bar{V}$ satisfy $\Phi\left(\bar{u}^{-}, \bar{u}^{+}, \bar{V}\right)=0$, and conditions (3.5), (3.6) and (3.11). Moreover $\bar{q}^{-} / \bar{\rho}^{-} \neq \bar{V} \neq \bar{q}^{+} / \bar{\rho}^{+}$, i.e., there is transfer of mass between the two sides of the solid body.

In practical applications it is often unavoidable to consider the presence of, at least, one end of the tube. This leads to study the following initial-boundary data for a balance law, coupled with an ordinary differential equation, defined for $x>0$ with a no flow condition at $x=0$ :

$$
\left\{\begin{array}{l}
\partial_{t} \rho+\partial_{x} q=0, \\
\partial_{t} q+\partial_{x} P=-\rho g \sin \vartheta-v_{F}(\rho, q), \\
\ddot{X}=-g \sin \vartheta-\frac{1}{m}(\Delta(A P)-\dot{X} \Delta(A q))-v_{S}(\dot{X}), \\
\Delta(A q)-\dot{X} \Delta(A \rho)=0, \\
\Delta(A F)-\dot{X} \Delta(A E)=\dot{X}(\Delta(A P)-\dot{X} \Delta(A q))-v_{I}(q / \rho, \dot{X}), \\
q(t, 0+)=0 .
\end{array}\right.
$$


Note that Example 2.2, if $X_{\boldsymbol{o}}>0$, provides a stationary solution to (3.12). For the case of two boundaries, we refer to [5].

From a rigorously analytical point of view, problem (3.12) does not fit in the framework in [4], due to the presence of the boundary condition at $x=0$. Hence a result analogous to Theorem 3.6, ensuring the well posedness of solutions, can not be proved relying on the cited literature. However the analytical results in $[4,9]$ can be easily merged, obtaining a theorem which includes also the presence of a boundary, hence proving the well posedness of the Cauchy problem for (3.12). This merely technical improvement is out of the scope of the present paper.

\section{Numerical examples}

This section is devoted to numerical integrations of (2.14) and (3.12). The balance laws are solved by means of a wave-propagation method for a moving mesh, see [12]. The ordinary differential equation and the source term are integrated using a two stages Runge-Kutta method. The two systems are coupled using the Strang splitting [16, § 17.4] in order to maintain the second order accuracy. The time steps are chosen according to a CFL number of 0.9. Particular care was taken to ensure the conservation of the conserved quantities and the matching of the trace conditions at the solid body.

\subsection{Fluid flowing past a moving body}

We consider a rigid body moving in a tube filled with a compressible fluid. Initially, the body travels at the same speed as the surrounding fluid. Hence, the framework in [4] applies. At a later time, a relatively strong shock hits the body, accelerating it. As a result, the body starts moving at a speed different from that of the fluid, thus allowing transfer of mass between its two sides. This latter behavior is not allowed in [4], but it is comprised in the present framework based on (2.14).
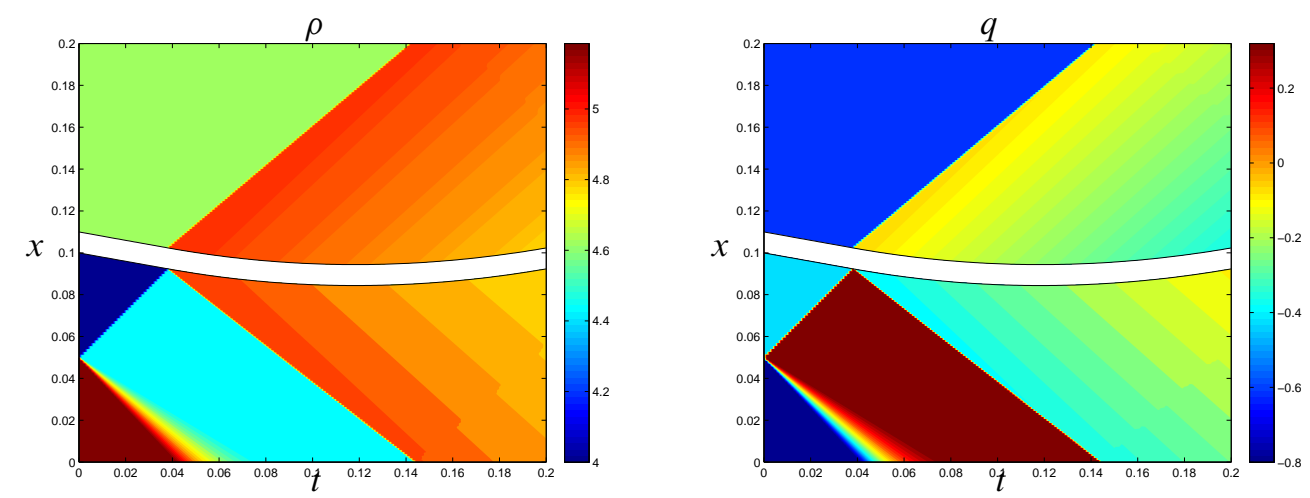

FIG. 2. Integration of (2.14), (2.3), (2.13), (4.1). Left, the density $\rho$ and, right, the flow $q$ as functions of $t$ (on the horizontal axis) and $x$ (on the vertical axis). The position of the solid body is indicated by the white gap. 
More precisely, we consider (2.14), with the pressure law (2.3), with all the friction terms (2.13) set to 0 and with the parameters

$$
\begin{aligned}
\sigma=1 \frac{\mathrm{m}}{\mathrm{s}}, & g=9.81 \frac{\mathrm{m}}{\mathrm{s}^{2}}, \quad \vartheta=0, \\
A^{-}=1 \mathrm{~m}^{2}, & A^{+}=0.5 \mathrm{~m}^{2}, \quad m=1 \mathrm{~kg} .
\end{aligned}
$$

We choose the following initial data:

$$
\rho(0, x)=\left\{\begin{array}{lll}
6 \frac{\mathrm{kg}}{\mathrm{m}} & x<0.05 \mathrm{~m}, & q(0, x)=-0.2 \frac{\mathrm{m}}{\mathrm{s}} \rho(0, x), \\
2 \frac{\mathrm{kg}}{\mathrm{m}} & x \in[0.05 \mathrm{~m}, 0.1 \mathrm{~m}], & X(0)=0.1 \mathrm{~m}, \\
4 \frac{\mathrm{kg}}{\mathrm{m}} & x>0.1 \mathrm{~m}, & \dot{X}(0)=-0.2 \frac{\mathrm{m}}{\mathrm{s}} .
\end{array}\right.
$$

The numerical integration of (2.14), (2.3), (2.13), (4.1), obtained with $N=2000$ grid cells up to the time $T=0.2 \mathrm{~s}$, is shown in Figures 2 and 3. At the beginning, the solid body and the fluid have the same speed, see Figure 3. At this stage, the whole system can be described by the framework in [3, 4]. The Riemann problem at $t=0 \mathrm{~s}, x=0.05 \mathrm{~m}$ yields a 2-shock moving upwards, which hits the solid body at about $t=0.038$, see Figure 2. As a result of this interaction, the solid body acceleration $\ddot{X}$ suffers a discontinuity, as also the traces of the fluid speed at the solid body. From that instant on, the three speeds are different, see Figure 3 right, but related by the conservation of mass through the ratio of the sections $A^{+} / A^{-}$. Remarkably, the numerical integration captures the fact that if two of these speeds are equal at some time $\bar{t}$, then also the third speed must attain this common value at the same time, see Figure 3, right, at about $\bar{t}=0.12 \mathrm{~s}$. Due to the absence of friction, system (2.14), (2.3), (2.13), (4.1) is Galileian invariant. Hence, the common value of the three speeds, in this case $v \approx 0 \mathrm{~m} / \mathrm{s}$, is not relevant.

Remark that at the interaction between the shock and the solid body [4, Proposition 3.1] may not be applied: when this large shock hits the solid body, the speed of the fluid above and below the fluid become different from that of the solid body.
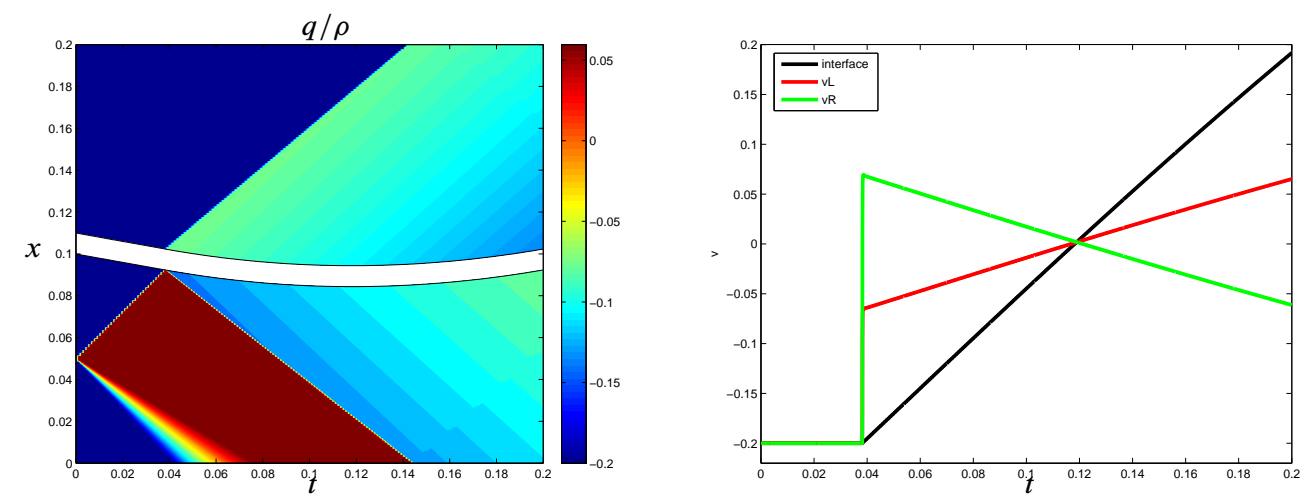

FIG. 3. Integration of (2.14), (2.3), (2.13), (4.1). Left, the speed $v$ as a function of $t$ (on the horizontal axis) and $x$ (on the vertical axis). The position of the solid body is indicated by the white gap. Right, the black line is the speed of the solid body, the red, respectively green, line is the trace of the fluid speed at the solid body from below, respectively from above. 


\subsection{A ball bouncing on a shock in a compressible fluid}

In the following we consider a vertical cylinder filled with a compressible fluid and containing a ball falling downwards. The cylinder has a fixed cross section, is closed at the bottom end $x=0 \mathrm{~m}$ and is open at the top. The gas within the cylinder is characterized by the pressure law (2.3), the friction terms (2.13) and the following parameters are chosen:

$$
\begin{aligned}
& \sigma=15 \frac{\mathrm{m}}{\mathrm{s}} \quad g=9.81 \frac{\mathrm{m}}{\mathrm{s}^{2}} \quad \vartheta=\pi / 2 \\
& \tilde{v}_{F}=10^{-8} \frac{1}{\mathrm{~m}} \quad \tilde{v}_{S}=10^{-2} \frac{1}{\mathrm{~s}} \quad \tilde{v}_{I}=5 \frac{\mathrm{m}^{2} \mathrm{~kg}}{\mathrm{~s}} \\
& m=0.004 \mathrm{~kg}
\end{aligned}
$$

We choose the following initial data:

$$
\rho(0, x)=1 / 225 \frac{\mathrm{kg}}{\mathrm{m}}, \quad q(0, x)=0 \frac{\mathrm{kg}}{\mathrm{s}}, \quad X(0)=0.35 \mathrm{~m}, \quad \dot{X}(0)=-2.5 \frac{\mathrm{m}}{\mathrm{s}} .
$$

The numerical integration, obtained with $N=2000$ grid cells up to the time $T=0.3 \mathrm{~s}$, is shown in figures 4 and 5. At the beginning the ball falls at a relatively high speed. Hence, it compresses the gas and generates a compression wave of higher density propagating downwards. During its way down, the ball is slowed down due to several effects. As the surrounding fluid is almost at rest, the friction decreases the ball's speed on the time interval [0 s, $0.04 \mathrm{~s}]$. At the same time, the compression wave caused by the ball focuses in a shock, reaches the bottom at about $t=0.02 \mathrm{~s}$, bounces back and at about $t=0.04 \mathrm{~s}$ hits the ball, causing a discontinuity in its acceleration $\ddot{X}$. At this interaction the shock is mainly reflected, but a smaller part passes the ball and later exits the integration domain. The reflected part of the shock is mostly trapped between the bottom and the moving ball. In fact, at any interaction between the ball and the shocks, only a small part of the wave is transmitted while most of the shock is reflected and continues bouncing back and forth between the ball and the bottom. The fact that the shock strength diminishes with time is confirmed also in Figure 5, right: the momentum transfer from the shock to the ball due to the interaction with the shock is less and less evident as the number of bounces increases. Thus, the ball is pushed upward
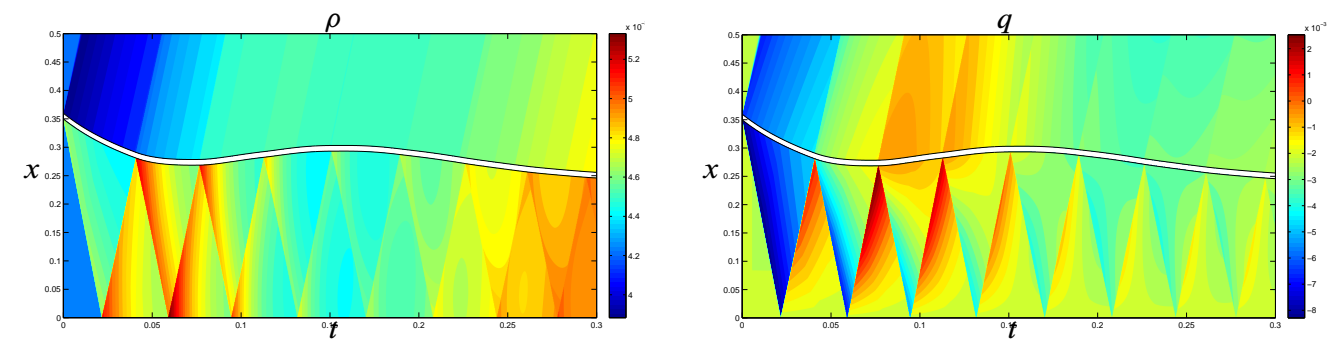

FIG. 4. A ball falling in a gas, i.e., integration of (3.12), (2.3), (2.13), (4.3) with initial datum (4.4), see also Figure 5. Left, the density $\rho$ and, right, the flow $q$ as functions of $t$ (on the horizontal axis) and $x$ (on the vertical axis). The position of the ball is indicated by the white gap. The moving ball causes a compression wave that becomes a shock, bounces repeatedly between the bottom and the ball, slowing it down. 

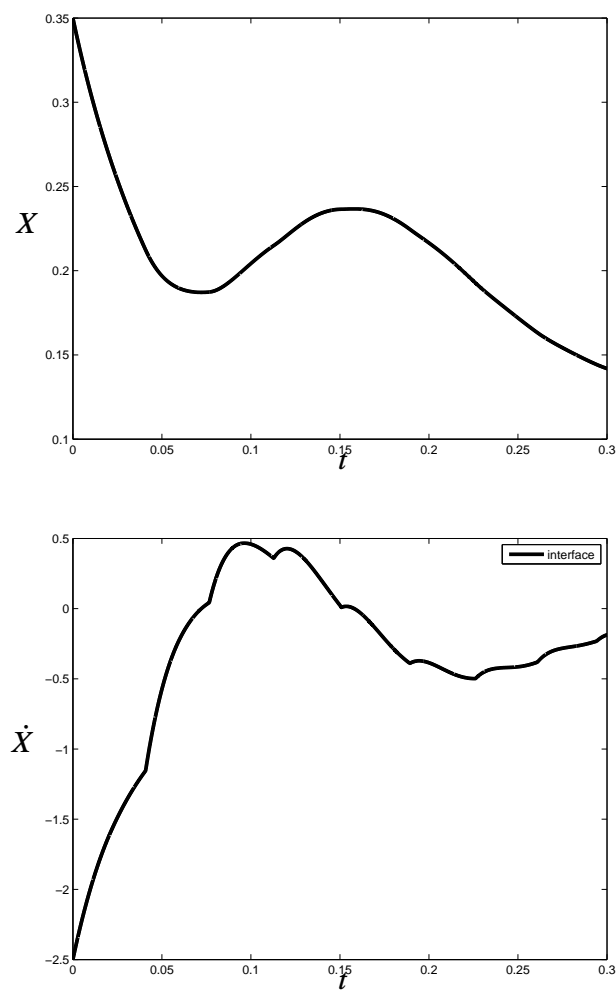

FIG. 5. Integration of (3.12), (2.3), (2.13), (4.3) with initial datum (4.4), see also Figure 4. Left, the height $X$ and, right, the velocity $\dot{X}$ of the ball falling in the gas. Note the discontinuities in $\ddot{X}$ due to the shocks hitting the ball: their size diminishes with time due to the decreasing size of the bouncing shock.

each time it is hit by the wave but, asymptotically, it will reach the bottom, since the lower fluid is allowed to pass above the ball.

We remark the evident analogy with the experimental findings in [1], where a ball falling in a stratified fluid is observed to follow very similar trajectories, see in particular [1, figures 4,5 and 6].

\section{Technical details}

Recall the sound speed $c$, the eigenvalues $\lambda_{1,2}$, the eigenvectors $r_{1,2}$ and the Riemann coordinates $v_{1,2}$ referred to the $p$-system (2.1):

$$
\begin{aligned}
& \lambda_{1}(\rho, q)=\frac{q}{\rho}-c(\rho) \\
& \lambda_{2}(\rho, q)=\frac{q}{\rho}+c(\rho) \\
& c(\rho)=\sqrt{p^{\prime}(\rho)} \quad r_{1}(\rho, q)=\left[\begin{array}{c}
\rho \\
q-\rho c(\rho)
\end{array}\right] \\
& r_{2}(\rho, q)=\left[\begin{array}{c}
\rho \\
q+\rho c(\rho)
\end{array}\right] \\
& v_{1}=\frac{q}{\rho}+\int_{\rho_{*}}^{\rho} \frac{c(r)}{r} \mathrm{~d} r \\
& v_{2}=\frac{q}{\rho}-\int_{\rho_{*}}^{\rho} \frac{c(r)}{r} \mathrm{~d} r
\end{aligned}
$$


where $\rho_{*}>0$ is a fixed reference density.

Lemma 5.1 Assume (p) holds. Let $E$ and $F$ be the functions defined in (2.4). Then $E$ is a convex function and an entropy for the $p$-system (2.5) with entropy flux $F$.

Proof. The Hessian matrix of $E$ is given by

$$
D^{2} E(\rho, q)=\left[\begin{array}{cc}
\frac{q^{2}}{\rho^{3}}+\frac{p^{\prime}(\rho)}{\rho} & -\frac{q}{\rho^{2}} \\
-\frac{q}{\rho^{2}} & \frac{1}{\rho}
\end{array}\right],
$$

which, by (p), is a semidefinite positive matrix. This proves that $E$ is a convex function. Moreover

$$
\begin{aligned}
& D E(\rho, q)=\left[\begin{array}{ll}
-\frac{q^{2}}{2 \rho^{2}}+\int_{\rho_{*}}^{\rho} \frac{p(r)}{r^{2}} \mathrm{~d} r+\frac{p(\rho)}{\rho} & \frac{q}{\rho}
\end{array}\right], \\
& D F(\rho, q)=\left[\begin{array}{ll}
-\frac{q^{3}}{\rho^{3}}+\frac{q p^{\prime}(\rho)}{\rho} & \frac{3 q^{2}}{2 \rho^{2}}+\int_{\rho_{*}}^{\rho} \frac{p(r)}{r^{2}} \mathrm{~d} r+\frac{p(\rho)}{\rho}
\end{array}\right],
\end{aligned}
$$

while the Jacobian matrix of the flux function for (2.5) is given by

$$
D f(\rho, q)=\left[\begin{array}{cc}
0 & 1 \\
-\frac{q^{2}}{\rho^{2}}+p^{\prime}(\rho) & \frac{2 q}{\rho}
\end{array}\right] .
$$

It is immediate to show that $D E \cdot D f=D F$, hence $E$ and $F$ provide an entropy and entropy flux pair for (2.5).

Lemma 5.2 Let $p$ satisfy (p). Then, for any $g>0, \vartheta \in\left[0, \frac{\pi}{2}\right], v \in \mathbb{R}$ and $\rho_{o} \in \stackrel{\mathbb{R}}{ }^{+}$, the Cauchy Problem

$$
\left\{\begin{array}{l}
\partial_{x}\left(\rho v^{2}+p(\rho)\right)=-\rho g \sin \vartheta \\
\rho(0)=\rho_{o}
\end{array}\right.
$$

admits a unique solution defined on all $\mathbb{R}$. Moreover:

1. If $\vartheta=0$, then $\rho(x)=\rho_{o}$ for every $x \in \mathbb{R}$.

2. If $v \neq 0$, then $\rho(x)>0$ for every $x \in \mathbb{R}$.

3. If $\vartheta>0$ and $v=0$, then $\rho(x)>0$ for every $x \in \mathbb{R}$ if and only if $\int_{0}^{\rho_{o}} \frac{p^{\prime}(\rho)}{\rho} \mathrm{d} \rho=+\infty$.

In particular, if (2.2) holds, then there exists $\bar{x}>0$ such that $\rho(x)>0$ for every $x<\bar{x}$ and $\rho(x)=0$ for every $x \geqslant \bar{x}$. Otherwise, if (2.3) holds, then $\rho(x)>0$ for every $x \in \mathbb{R}$.

4. If (2.2) holds, then, for every $x \in \mathbb{R}$ such that $\rho(x)>0$, we have

$$
v^{2} \log (\rho(x))+\frac{p_{o} \gamma}{\rho_{o}^{\gamma}(\gamma-1)} \rho^{\gamma-1}(x)=-x g \sin \vartheta+v^{2} \log \left(\rho_{o}\right)+\frac{p_{o} \gamma}{\rho_{o}^{\gamma}(\gamma-1)} \rho_{o}^{\gamma-1}
$$

5. If (2.3) holds, then, for every $x \in \mathbb{R}$, we have $\rho(x)=\rho_{o} \exp \left(-\frac{g \sin \vartheta}{v^{2}+\sigma^{2}} x\right)$.

Proof. Note that the differential equation in (5.2) can be written in the form

$$
\left[v^{2}+p^{\prime}(\rho(x))\right] \rho^{\prime}(x)=-\rho(x) g \sin \vartheta,
$$


The solution is strictly monotone increasing in its domain of existence. If $v \neq 0$, then, by (p), the Cauchy theorem for ordinary differential equations applies providing global existence and uniqueness of a solution to (5.2). Moreover, by a comparison principle, one easily deduces that $\rho(x)>0$ for every $x \in \mathbb{R}$.

Assume now that $v=0$. If $\vartheta=0$, then clearly the unique solution is constant. Suppose that $\vartheta>0$. Then, in a neighborhood of $x=0$, the solution to (5.2) exists and remains positive. Then the differential equation in (5.2) can be written in the form

$$
\frac{p^{\prime}(\rho(x))}{\rho(x)} \rho^{\prime}(x)=-g \sin \vartheta .
$$

By (p), we easily deduce that $\int_{\rho_{o}}^{+\infty} \frac{p^{\prime}(\rho)}{\rho} \mathrm{d} \rho=+\infty$ hence there is no blowup in finite time, i.e., $\rho(x)$ exists for every $x \in \mathbb{R}$. Moreover, the integral

$$
\int_{0}^{\rho_{o}} \frac{p^{\prime}(\rho)}{\rho} \mathrm{d} \rho
$$

could be finite or $+\infty$. If it is equal to $+\infty$, then the solution asymptotically tends to 0 when $x \rightarrow+\infty$. If the integral in (5.3) is finite, then there exists $\bar{x}>0$ such that $\rho(\bar{x})=0$ and $\rho(x)>0$ for every $x<\bar{x}$. Note also that $\rho$ can be prolonged in a unique way after $\bar{x}$ (by $\rho(x)=0$ ). The last statements of the lemma easily follow.

Proof of Lemma 3.3. First observe that the hypotheses of the Lemma imply that the quantities in (2.7) are well defined and finite. Indeed, it is clear that $m(t)$ and $Q(t)$ are well defined and finite. As regards $\varepsilon(t)$, the hypotheses on the pressure law $p$ imply that there exist a constant $K>0$ and $\bar{\rho}>0$ such that $p(\rho) \leqslant K \rho$ for every $0<\rho<\bar{\rho}$. From this inequality and from the hypotheses of the lemma easily follows that the energy $E(\rho(t, x), q(t, x)) \in \mathbf{L}^{\mathbf{1}}(\mathbb{R})$ for every $t \geqslant 0$, proving that $\varepsilon(t)$ is also well defined and finite. The conservation of mass is a standard property of conservation laws, see [11].

Note that the regularity of $u$ prescribed in Definition 3.1 does not ensure the differentiability of $Q$ and $\varepsilon$. Therefore for $T>0$, we prove that

$$
\int_{0}^{T} Q(t) \varphi^{\prime}(t) \mathrm{d} t=\int_{0}^{T}\left(\int_{\mathbb{R}} A(t, x) v_{F}(\rho(t, x), q(t, x)) \mathrm{d} x+m v_{S}(\dot{X}(t))+m g \sin \vartheta\right) \varphi(t) \mathrm{d} t
$$

for any $\varphi \in \mathbf{C}_{\mathbf{c}}^{\mathbf{1}}(] 0, T[; \mathbb{R})$ and

$$
\begin{array}{r}
\int_{0}^{T} \mathcal{E}(t) \varphi^{\prime}(t) \mathrm{d} t \geqslant \int_{0}^{T}\left[\int_{\mathbb{R}}\left(A(t, x) q(t, x) v_{F}(\rho(t, x), q(t, x))+v_{I}\left(\frac{q(t, x)}{\rho(t, x)}, \dot{X}(t)\right)\right) \mathrm{d} x\right. \\
\left.+m v_{S}(\dot{X}) \dot{X}(t)\right] \varphi(t) \mathrm{d} t
\end{array}
$$

for any $\varphi \in \mathbf{C}_{\mathbf{c}}^{\mathbf{1}}(] 0, T\left[; \mathbb{R}^{+}\right)$. Fix an increasing sequence of functions $\vartheta_{n} \in \mathbf{C}_{\mathbf{c}}^{\mathbf{1}}\left(\stackrel{\circ}{ }^{-} ;[0,1]\right)$ such that $\vartheta_{n}(x) \rightarrow 1$ for a.e. $x \in \mathbb{R}^{-}$. Define

$$
Q_{n}(t)=m \dot{X}(t)+A^{-} \int_{-\infty}^{X(t)} \vartheta_{n}(x-X(t)) q(t, x) \mathrm{d} x+A^{+} \int_{X(t)}^{+\infty} \vartheta_{n}(X(t)-x) q(t, x) \mathrm{d} x .
$$


Note that, for a.e. $t \in[0, T], Q_{n}(t) \rightarrow \mathcal{Q}(t)$ as $n \rightarrow+\infty$. We have

$$
\begin{aligned}
\int_{0}^{T} Q_{n}(t) \varphi^{\prime}(t) \mathrm{d} t=m \int_{0}^{T} \dot{X}(t) \varphi^{\prime}(t) \mathrm{d} t & +A^{-} \int_{0}^{T} \int_{-\infty}^{X(t)} \varphi^{\prime}(t) \vartheta_{n}(x-X(t)) q(t, x) \mathrm{d} x \mathrm{~d} t \\
& +A^{+} \int_{0}^{T} \int_{X(t)}^{+\infty} \varphi^{\prime}(t) \vartheta_{n}(X(t)-x) q(t, x) \mathrm{d} x \mathrm{~d} t .
\end{aligned}
$$

By Definition 3.1, we obtain that

$$
\begin{aligned}
\int_{0}^{T} Q_{n}(t) \varphi^{\prime}(t) \mathrm{d} t & =-m \int_{0}^{T} \ddot{X}(t) \varphi(t) \mathrm{d} t \\
& -A^{-} \int_{0}^{T} \int_{-\infty}^{X(t)} \varphi(t) \vartheta_{n}^{\prime}(x-X(t))\left(\frac{q^{2}(t, x)}{\rho(t, x)}+p(\rho(t, x))\right) \mathrm{d} x \mathrm{~d} t \\
& +A^{-} \int_{0}^{T} \int_{-\infty}^{X(t)} \varphi(t) \vartheta_{n}(x-X(t))\left(\rho(t, x) g \sin \vartheta+v_{F}(\rho(t, x), q(t, x))\right) \mathrm{d} x \mathrm{~d} t \\
& +A^{-} \int_{0}^{T} \int_{-\infty}^{X(t)} \varphi(t) \vartheta_{n}^{\prime}(x-X(t)) \dot{X}(t) q(t, x) \mathrm{d} x \mathrm{~d} t \\
& +A^{+} \int_{0}^{T} \int_{X(t)}^{+\infty} \varphi(t) \vartheta_{n}^{\prime}(X(t)-x)\left(\frac{q^{2}(t, x)}{\rho(t, x)}+p(\rho(t, x))\right) \mathrm{d} x \mathrm{~d} t \\
& +A^{+} \int_{0}^{T} \int_{X(t)}^{+\infty} \varphi(t) \vartheta_{n}(X(t)-x)\left(\rho(t, x) g \sin \vartheta+v_{F}(\rho(t, x), q(t, x))\right) \mathrm{d} x \mathrm{~d} t \\
& -A^{+} \int_{0}^{T} \int_{X(t)}^{+\infty} \varphi(t) \vartheta_{n}^{\prime}(X(t)-x) \dot{X}(t) q(t, x) \mathrm{d} x \mathrm{~d} t
\end{aligned}
$$

Passing to the limit as $n \rightarrow+\infty$ we have

$$
\begin{aligned}
\int_{0}^{T} Q(t) \varphi^{\prime}(t) \mathrm{d} t & =-m \int_{0}^{T} \ddot{X}(t) \varphi(t) \mathrm{d} t \\
& +\int_{0}^{T} \varphi(t)[-\Delta(A P)(t)+\dot{X}(t) \Delta(A q)(t)] \mathrm{d} t \\
& +A^{-} \int_{0}^{T} \int_{-\infty}^{X(t)} \varphi(t)\left(\rho(t, x) g \sin \vartheta+v_{F}(\rho(t, x), q(t, x))\right) \mathrm{d} x \mathrm{~d} t \\
& +A^{+} \int_{0}^{T} \int_{X(t)}^{+\infty} \varphi(t)\left(\rho(t, x) g \sin \vartheta+v_{F}(\rho(t, x), q(t, x))\right) \mathrm{d} x \mathrm{~d} t
\end{aligned}
$$

and, using (2.14) and the assumptions on $u_{l}$ and $u_{r}$, we get

$$
\begin{array}{r}
\int_{0}^{T} Q(t) \varphi^{\prime}(t) \mathrm{d} t=g \sin \vartheta \int_{0}^{T} \varphi(t)\left(m+\int_{\mathbb{R}} A(t, x) \rho(t, x) \mathrm{d} x\right) \mathrm{d} t+m \int_{0}^{T} \varphi(t) v_{S}(\dot{X}(t)) \mathrm{d} t \\
+\int_{0}^{T} \int_{\mathbb{R}} \varphi(t) A(t, x) v_{F}(\rho(t, x), q(t, x)) \mathrm{d} x \mathrm{~d} t
\end{array}
$$


proving the second statement of Lemma 3.3.

Let us consider now the inequality satisfied by the distributional derivative of the total energy $\varepsilon$. Fix an increasing sequence of functions $\vartheta_{n} \in \mathbf{C}_{\mathbf{c}}^{\mathbf{1}}\left(\stackrel{\circ}{\mathbb{R}}^{-} ;[0,1]\right)$ such that $\vartheta_{n}(x) \rightarrow 1$ for a.e. $x \in \mathbb{R}^{-}$. Note that the hypotheses imply that

$$
\lim _{x \rightarrow \pm \infty} E(\rho(t, x), q(t, x))=\lim _{x \rightarrow \pm \infty} F(\rho(t, x), q(t, x))=0
$$

for every $t \geqslant 0$. Define

$$
\begin{aligned}
& \varepsilon_{n}(t)= m g(t) \\
& \sin \vartheta+\frac{1}{2} m \dot{X}^{2}(t) \\
& \quad+A^{-} \int_{-\infty}^{X(t)} \vartheta_{n}(x-X(t))(E(\rho(t, x), q(t, x))+\rho(t, x) x g \sin \vartheta) \mathrm{d} x \\
& \quad+A^{+} \int_{X(t)}^{+\infty} \vartheta_{n}(X(t)-x)(E(\rho(t, x), q(t, x))+\rho(t, x) x g \sin \vartheta) \mathrm{d} x .
\end{aligned}
$$

Note that, for a.e. $t \in[0, T], \varepsilon_{n}(t) \rightarrow \varepsilon(t)$ as $n \rightarrow+\infty$. We have

$$
\begin{aligned}
\int_{0}^{T} \varphi^{\prime}(t) \varepsilon_{n}(t) \mathrm{d} t=\int_{0}^{T} & \varphi^{\prime}(t)\left(m g X(t) \sin \vartheta+\frac{1}{2} m \dot{X}^{2}(t)\right) \mathrm{d} t \\
& +A^{-} \int_{0}^{T} \int_{-\infty}^{X(t)} \varphi^{\prime}(t) \vartheta_{n}(x-X(t)) E(\rho(t, x), q(t, x)) \mathrm{d} x \mathrm{~d} t \\
& +A^{-} \int_{0}^{T} \int_{-\infty}^{X(t)} \varphi^{\prime}(t) \vartheta_{n}(x-X(t)) \rho(t, x) g x \sin \vartheta \mathrm{d} x \mathrm{~d} t \\
& +A^{+} \int_{0}^{T} \int_{X(t)}^{+\infty} \varphi^{\prime}(t) \vartheta_{n}(X(t)-x) E(\rho(t, x), q(t, x)) \mathrm{d} x \mathrm{~d} t \\
& +A^{+} \int_{0}^{T} \int_{X(t)}^{+\infty} \varphi^{\prime}(t) \vartheta_{n}(X(t)-x) \rho(t, x) g x \sin \vartheta \mathrm{d} x \mathrm{~d} t
\end{aligned}
$$

By Lemma 5.1 the couple $(E, F)$ provides a convex entropy-entropy flux pair for the $p$ system (2.5), hence, by Definition 3.1, we obtain

$$
\begin{aligned}
& \int_{0}^{T} \varphi^{\prime}(t) \varepsilon_{n}(t) \mathrm{d} t \\
& \geqslant \int_{0}^{T} \varphi^{\prime}(t)\left(m g X(t) \sin \vartheta+\frac{1}{2} m \dot{X}^{2}(t)\right) \mathrm{d} t \\
& -A^{-} \int_{0}^{T} \int_{-\infty}^{X(t)} \varphi(t) \vartheta_{n}^{\prime}(x-X(t)) F(\rho(t, x), q(t, x)) \mathrm{d} x \mathrm{~d} t \\
& +A^{-} \int_{0}^{T} \int_{-\infty}^{X(t)} \varphi(t) \vartheta_{n}(x-X(t))\left[g \sin \vartheta q(t, x)+v_{F}(\rho(t, x), q(t, x)) \frac{q(t, x)}{\rho(t, x)}\right] \mathrm{d} x \mathrm{~d} t \\
& +A^{-} \int_{0}^{T} \int_{-\infty}^{X(t)} \varphi(t) \vartheta_{n}^{\prime}(x-X(t)) \dot{X}(t) E(\rho(t, x), q(t, x)) \mathrm{d} x \mathrm{~d} t
\end{aligned}
$$




$$
\begin{aligned}
& -A^{-} g \sin \vartheta \int_{0}^{T} \int_{-\infty}^{X(t)} \varphi(t) \vartheta_{n}^{\prime}(x-X(t)) x q(t, x) \mathrm{d} x \mathrm{~d} t \\
& -A^{-} g \sin \vartheta \int_{0}^{T} \int_{-\infty}^{X(t)} \varphi(t) \vartheta_{n}(x-X(t)) q(t, x) \mathrm{d} x \mathrm{~d} t \\
& +A^{-} g \sin \vartheta \int_{0}^{T} \int_{-\infty}^{X(t)} \varphi(t) \vartheta_{n}^{\prime}(x-X(t)) \dot{X}(t) x \rho(t, x) \mathrm{d} x \mathrm{~d} t \\
& +A^{+} \int_{0}^{T} \int_{X(t)}^{+\infty} \varphi(t) \vartheta_{n}^{\prime}(X(t)-x) F(\rho(t, x), q(t, x)) \mathrm{d} x \mathrm{~d} t \\
& +A^{+} \int_{0}^{T} \int_{X(t)}^{+\infty} \varphi(t) \vartheta_{n}(X(t)-x)\left[g \sin \vartheta q(t, x)+v_{F}(\rho(t, x), q(t, x)) \frac{q(t, x)}{\rho(t, x)}\right] \mathrm{d} x \mathrm{~d} t \\
& -A^{+} \int_{0}^{T} \int_{X(t)}^{+\infty} \varphi(t) \vartheta_{n}^{\prime}(X(t)-x) \dot{X}(t) E(\rho(t, x), q(t, x)) \mathrm{d} x \mathrm{~d} t \\
& +A^{+} g \sin \vartheta \int_{0}^{T} \int_{X(t)}^{+\infty} \varphi(t) \vartheta_{n}^{\prime}(X(t)-x) x q(t, x) \mathrm{d} x \mathrm{~d} t \\
& -A^{+} g \sin \vartheta \int_{0}^{T} \int_{X(t)}^{+\infty} \varphi(t) \vartheta_{n}(X(t)-x) q(t, x) \mathrm{d} x \mathrm{~d} t \\
& -A^{+} g \sin \vartheta \int_{0}^{T} \int_{X(t)}^{+\infty} \varphi(t) \vartheta_{n}^{\prime}(X(t)-x) \dot{X}(t) x \rho(t, x) \mathrm{d} x \mathrm{~d} t .
\end{aligned}
$$

Passing to the limit as $n \rightarrow+\infty$, we have

$$
\begin{aligned}
\int_{0}^{T} \varphi^{\prime}(t) \varepsilon(t) \mathrm{d} t \geqslant \int_{0}^{T} & \varphi^{\prime}(t)\left(m g X(t) \sin \vartheta+\frac{1}{2} m \dot{X}^{2}(t)\right) \mathrm{d} t \\
& +\int_{0}^{T} \varphi(t)(-\Delta(A F(t))+\dot{X}(t) \Delta(A E(t))) \mathrm{d} t \\
& +g \sin \vartheta \int_{0}^{T} \varphi(t) X(t)(-\Delta(A q(t))+\dot{X}(t) \Delta(A \rho(t))) \mathrm{d} t \\
& +\int_{0}^{T} \int_{\mathbb{R}} \varphi(t) A(t, x) v_{F}(\rho(t, x), q(t, x)) \frac{q(t, x)}{\rho(t, x)} \mathrm{d} x \mathrm{~d} t .
\end{aligned}
$$

Now, using the relations (2.14), we deduce that

$$
\begin{aligned}
\int_{0}^{T} \varphi^{\prime}(t) \varepsilon(t) \mathrm{d} t \geqslant \int_{0}^{T} \varphi(t) v_{I}\left(\frac{q(t, x)}{\rho(t, x)}, \dot{X}(t)\right) \mathrm{d} t+m \int_{0}^{T} \varphi(t) \dot{X}(t) v_{S}(\dot{X}(t)) \mathrm{d} t \\
\quad+\int_{0}^{T} \int_{\mathbb{R}} \varphi(t) A(t, x) v_{F}(\rho(t, x), q(t, x)) \frac{q(t, x)}{\rho(t, x)} \mathrm{d} x \mathrm{~d} t
\end{aligned}
$$

which proves the Lemma.

Proof of Proposition 3.5. In a neighborhood of $\left(u^{-}, u^{+}\right) \in\left(\mathbb{R}^{+} \times \mathbb{R}\right)^{2}$, introduce the function $\tilde{\Phi}$ as the expression of $\Phi$ in (3.4) in the Riemann coordinates $v^{ \pm}$defined as in (5.1). The condition 
$\Phi\left(u^{-}, u^{+}, V\right)=0$ is then rewritten as

$$
\tilde{\Phi}\left(v^{-}, v^{+}, V\right)=0 .
$$

In a neighborhood of $\left(\bar{v}^{-}, \bar{v}^{+}, V\right)$, the Implicit Function Theorem can be applied to (5.4), so that it is locally equivalent to

$$
v_{1}^{-}=\Psi_{1}\left(v_{2}^{-}, v_{1}^{+}, V\right) \quad \text { and } \quad v_{2}^{+}=\Psi_{2}\left(v_{2}^{-}, v_{1}^{+}, V\right)
$$

for suitable smooth functions $\Psi_{1}, \Psi_{2}$, since

$$
\begin{aligned}
D_{\left(v_{1}^{-}, v_{2}^{+}\right)} \tilde{\Phi}\left(\bar{v}^{-}, \bar{v}^{+}, \bar{V}\right) & =D_{\left(u^{-}, u^{+}\right)} \Phi\left(\bar{u}^{-}, \bar{u}^{+}, \bar{V}\right) \cdot D_{\left(v_{1}^{-}, v_{2}^{+}\right)}\left(\bar{u}^{-}, \bar{u}^{+}\right) \\
& =\left[\begin{array}{ll}
D_{u^{-}} \Phi\left(\bar{u}^{-}, \bar{u}^{+}, \bar{V}\right) & D_{u^{+}} \Phi\left(\bar{u}^{-}, \bar{u}^{+}, \bar{V}\right)
\end{array}\right]\left[\begin{array}{cc}
r_{1}\left(\bar{u}^{-}\right) & {\left[\begin{array}{l}
0 \\
0
\end{array}\right]} \\
{\left[\begin{array}{l}
0 \\
0
\end{array}\right]} & r_{2}\left(\bar{u}^{+}\right)
\end{array}\right] \\
& =\left[\begin{array}{ll}
D_{u^{-}} \Phi\left(\bar{u}^{-}, \bar{u}^{+}, \bar{V}\right) r_{1}\left(\bar{u}^{-}\right) & D_{u^{+}} \Phi\left(\bar{u}^{-}, \bar{u}^{+}, \bar{V}\right) r_{2}\left(\bar{u}^{+}\right)
\end{array}\right]
\end{aligned}
$$

and the latter matrix is invertible by (3.5).

To solve for $V$ each of the two the relations (5.5), we apply again the Implicit Function Theorem, computing

$$
\begin{aligned}
D_{V} \Psi\left(\bar{v}_{2}^{-}, \bar{v}_{1}^{+}, \bar{V}\right) & =-\left[D_{\left(v_{1}^{-}, v_{2}^{+}\right)} \tilde{\Phi}\left(\bar{v}^{-}, \bar{v}^{+}, \bar{V}\right)\right]^{-1} D_{V} \tilde{\Phi}\left(\bar{v}^{-}, \bar{v}^{+}, \bar{V}\right) \\
& =-\left[D_{u^{-}} \Phi\left(\bar{u}^{-}, \bar{u}^{+}, \bar{V}\right) r_{1}\left(\bar{u}^{-}\right) D_{u^{+}} \Phi\left(\bar{u}^{-}, \bar{u}^{+}, \bar{V}\right) r_{2}\left(\bar{u}^{+}\right)\right]^{-1} \\
& D_{V} \Phi\left(\bar{u}^{-}, \bar{u}^{+}, \bar{V}\right)
\end{aligned}
$$

and condition (3.6) allows to apply the Implicit Function Theorem proving that the relations (5.5) are logical equivalent to

$$
\tilde{b}^{-}\left(v_{1}^{-}, v_{2}^{-}, v_{1}^{+}\right)=V \quad \text { and } \quad \tilde{b}^{+}\left(v_{2}^{-}, v_{1}^{+}, v_{2}^{+}\right)=V .
$$

To complete the proof, let $b^{ \pm}$be the expression of $\tilde{b}^{ \pm}$in the Riemann coordinate and compute:

$$
\begin{aligned}
& \nabla_{u}-b^{-}\left(\bar{u}^{-}, \bar{u}^{+}\right) r_{1}\left(\bar{u}^{-}\right) \\
& =\nabla_{v}-\tilde{b}^{-}\left(\bar{v}^{-}, \bar{v}^{+}\right) D_{u^{-}} \bar{v}^{-} r_{1}\left(\bar{u}^{-}\right) \\
& =\left(\partial_{V} \Psi_{1}\left(\bar{v}_{2}^{-}, \bar{v}_{1}^{+}, \bar{V}\right)\right)^{-1}\left[\begin{array}{llll}
1 & \left.-\partial_{v_{2}} \Psi_{1}\left(\bar{v}_{2}^{-}, \bar{v}_{1}^{+}, \bar{V}\right)\right] & {\left[r_{1}\left(\bar{u}^{-}\right)\right.} & r_{2}\left(\bar{u}^{-}\right)
\end{array}\right]^{-1} r_{1}\left(\bar{u}^{-}\right)
\end{aligned}
$$

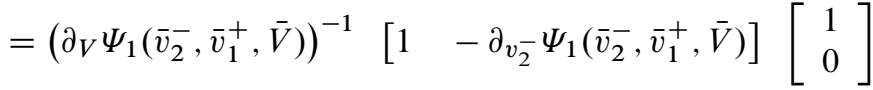

$$
\begin{aligned}
& =\left(\partial_{V} \Psi_{1}\left(\bar{v}_{2}^{-}, \bar{v}_{1}^{+}, \bar{V}\right)\right)^{-1} \\
& =-\left(\left[D_{u^{-}} \Phi\left(\bar{u}^{-}, \bar{u}^{+}, \bar{V}\right) r_{1}\left(\bar{u}^{-}\right) \quad D_{u^{+}} \Phi\left(\bar{u}^{-}, \bar{u}^{+}, \bar{V}\right) r_{2}\left(\bar{u}^{+}\right)\right]^{-1} D_{V} \Phi\left(\bar{u}^{-}, \bar{u}^{+}, \bar{V}\right)\right)_{1} \\
& \neq 0 \text {. }
\end{aligned}
$$

The case of $b^{+}$is entirely analogous. 
Proof of Lemma 3.7. Lengthy computations show that $\Phi\left(\bar{u}^{-}, \bar{u}^{+}, \bar{V}\right)=0$. Moreover the left hand side of (3.5) is equal to

$$
-\frac{1}{19}[\sqrt{\ln (10 / 9)}(405000 \sqrt{38} \ln (10 / 9)-42750 \sqrt{38})]+\frac{85500}{19} \ln (10 / 9) \sim 482,44 .
$$

Moreover the first condition in (3.6) is a fraction with numerator

$$
\begin{aligned}
& 32400 \ln ^{2}(5)+\sqrt{\ln (10 / 9)}(3240 \sqrt{38} \ln (10 / 9)-5 \sqrt{219488}) \\
& +(-129600 \ln (3)+64800 \ln (2)-722) \ln (5)+129600 \ln ^{2}(3) \\
& +(1444-129600 \ln (2)) \ln (3)+32400 \ln ^{2}(2)-722 \ln (2)-361
\end{aligned}
$$

and denominator

$$
\begin{aligned}
& \sqrt{\ln (10 / 9)}(34200 \sqrt{38} \ln (10 / 9)-95 \sqrt{219488}) \\
& -3078000 \ln ^{2}(5)+(12312000 \ln (3)-6156000 \ln (2)+646190) \ln (5)-12312000 \ln ^{2}(3) \\
& +(12312000 \ln (2)-1292380) \ln (3)-3078000 \ln ^{2}(2)+646190 \ln (2)-34295
\end{aligned}
$$

so that $(3.6)_{1} \sim 0,39$. The second condition in (3.6) is a fraction with the numerator

$$
\begin{aligned}
& 32400 \ln ^{2}(5)-\sqrt{\ln (10 / 9)}(3600 \sqrt{38} \ln (10 / 9)-9 \sqrt{219488}) \\
& -(129600 \ln (3)-64800 \ln (2)-722) \ln (5)+129600 \ln ^{2}(3) \\
& -(129600 \ln (2)+1444) \ln (3)+32400 \ln ^{2}(2)+722 \ln (2)-361 \sim 0,11
\end{aligned}
$$

and denominator

$$
\begin{aligned}
& \sqrt{\ln (10 / 9)}(34200 \sqrt{38}(\ln (5)-2 \ln (3)+\ln (2))-95 \sqrt{219488}) \\
& -3078000 \ln ^{2} 5+(12312000 \ln (3)-6156000 \ln (2)+646190) \ln (5)-12312000 \ln ^{2}(3) \\
& +(12312000 \ln (2)-1292380) \ln (3)-3078000 \ln ^{2}(2)+646190 \ln (2)-34295 \sim-393,81
\end{aligned}
$$

so that (3.6) $1 \sim-0,00027$. Finally condition (3.11) clearly holds, since $\bar{V} \sim-4,24$.

Lemma 5.3 Choose $p$ as in (2.3). Fix $g>0, \vartheta \in[0, \pi / 2]$. Let $A^{+}=A^{-}$in (2.11), $v_{F}(\rho, 0)=0$ and $v_{I} \equiv 0$ as in Example 2.1. Then, the initial datum $\left(u_{o}, X_{o}, V_{o}\right)$, where

$$
\begin{array}{ll}
\rho_{o}(x)=\bar{\rho} \exp \left(-\frac{g \sin \vartheta}{\sigma^{2}}(x-\bar{x})\right) & X_{o} \in \mathbb{R} \\
q_{o}(x)=0 & V_{o} \in \mathbb{R},
\end{array}
$$

satisfies (3.5), provided $V_{o} \neq \pm \sigma$ and $V \neq \frac{1}{2} \sigma \ln \left(\rho^{+} / \rho^{-}\right)$, but satisfies neither (3.6) ${ }_{1}$ nor (3.6) 2 . Proof. Concerning (3.5), lengthy but elementary computations lead to

$$
\operatorname{det}\left[D_{u^{-}} \Phi\left(\bar{u}^{-}, \bar{u}^{+}, \bar{V}\right) r_{1}\left(u^{-}\right) \quad D_{u^{+}} \Phi\left(\bar{u}^{-}, \bar{u}^{+}, \bar{V}\right) r_{2}\left(u^{+}\right)\right]=\sigma\left(V^{2}-\sigma^{2}\right)\left(2 V-\sigma \ln \frac{\rho^{+}}{\rho^{-}}\right)
$$

while both the right hand sides in (3.6) vanish. Indeed, with the notation in Proposition 3.5, $D_{V} \Phi\left(\bar{u}^{-}, \bar{u}^{+}, \bar{V}\right)=0$. 
Lemma 5.4 Choose $p$ as in (2.3) and $A$ as in (2.11) with $A^{ \pm}>0$. Fix $g>0, \vartheta \in[0, \pi / 2]$. Let $v_{F}=v_{S}=0$ as in Example 2.2 and $v_{I}(q / \rho, \dot{X})=\bar{v}_{I}(\dot{X}-q / \rho)^{2}$ for a positive $\bar{v}_{I}$. Assume $\rho^{ \pm}$ satisfy (2.17). Then, the initial datum $\left(u_{o}, X_{o}, V_{o}\right)$, where

$\rho_{o}(x)=\left\{\begin{array}{ll}\rho^{-} \exp \left(-\frac{g \sin \vartheta}{\sigma^{2}}\left(x-X_{o}\right)\right) & x<X_{o}, \\ \rho^{+} \exp \left(-\frac{g \sin \vartheta}{\sigma^{2}}\left(x-X_{o}\right)\right) & x>X_{o},\end{array} \quad q_{o}(x)=0, \quad X_{o} \in \mathbb{R}, \quad V_{o}=0\right.$,

satisfies (3.5) and (3.6), provided $\rho^{-} \neq \rho^{+}$.

Proof. Elementary but lengthy computations lead to

$$
\begin{aligned}
& \operatorname{det}\left[D_{u^{-}} \Phi\left(\bar{u}^{-}, \bar{u}^{+}, \bar{V}\right) r_{1}\left(u^{-}\right) \quad D_{u^{+}} \Phi\left(\bar{u}^{-}, \bar{u}^{+}, \bar{V}\right) r_{2}\left(u^{+}\right)\right]=A^{-} A^{+} \sigma^{4} \ln \left(\rho^{+} / \rho^{-}\right), \\
& \left(\left[D_{u^{-}} \Phi\left(\bar{u}^{-}, \bar{u}^{+}, \bar{V}\right) r_{1}\left(\bar{u}^{-}\right) \quad D_{u^{+}} \Phi\left(\bar{u}^{-}, \bar{u}^{+}, \bar{V}\right) r_{2}\left(\bar{u}^{+}\right)\right]^{-1} D_{V} \Phi\left(\bar{u}^{-}, \bar{u}^{+}, \bar{V}\right)\right)_{1}=\rho^{-} / \sigma, \\
& \left(\left[D_{u^{-}} \Phi\left(\bar{u}^{-}, \bar{u}^{+}, \bar{V}\right) r_{1}\left(\bar{u}^{-}\right) \quad D_{u^{+}} \Phi\left(\bar{u}^{-}, \bar{u}^{+}, \bar{V}\right) r_{2}\left(\bar{u}^{+}\right)\right]^{-1} D_{V} \Phi\left(\bar{u}^{-}, \bar{u}^{+}, \bar{V}\right)\right)_{2}=-\rho^{+} / \sigma,
\end{aligned}
$$

completing the proof.

\section{REFERENCES}

1. Abaid, N., Adalsteinsson, D., Agyapong, A., \& Mclaughlin, R. M. An internal splash: Levitation of falling spheres in stratified fluids. Phys. Fluids 16 (2004), 1567-1580. Zbl1186. 76012

2. Andreianov, B., Lagoutière, F., Seguin, N. \& Takahashi, T. Small solids in an inviscid fluid. Netw. Heterog. Media 5 (2010), 385-404. Zbl1262.35180 MR2670647

3. Borsche, R., Colombo, R. M. \& Garavello, M. On the coupling of systems of hyperbolic conservation laws with ordinary differential equations. Nonlinearity 23 (2010) 2749-2770. Zbl1205. 35170 MR2727168

4. Borsche, R., Colombo, R. M. \& Garavello, M. Mixed systems: ODEs-balance laws. J. Differential Equations 252 (2012), 2311-2338. Zbl1252. 35193 MR2860620

5. Borsche, R., Colombo, R. M. \& Garavello, M. Mixed systems with boundaries. Proceedings of Hyp2012, to appear.

6. BRESSAN, A. Hyperbolic systems of conservation laws, volume 20 of Oxford Lecture Series in Mathematics and its Applications. Oxford University Press, Oxford, 2000. The one-dimensional Cauchy problem. Zb10997.35002 MR1816648

7. Camassa, R., Falcon, C., Lin, J., Mclaughlin, R. M. \& Mykins, N. A first-principle predictive theory for a sphere falling through sharply stratified fluid at low Reynolds number. J. Fluid Mech. 664 (2010), 436-465. Zbl1221.76064 MR2747491

8. ČANIĆ, S. \& KIM, E. H.. Mathematical analysis of the quasilinear effects in a hyperbolic model blood flow through compliant axi-symmetric vessels. Math. Methods Appl. Sci. 26 (2003), 1161-1186. Zbl1141.76484 MR2002976

9. Colombo, R. M. \& Guerra, G.. On general balance laws with boundary. Journal of Differential Equations 248 (2010), 1017-1043. Zbl1196. 35136 MR2592880

10. Colombo, R. M., Guerra, G., Herty, M. \& Schleper, V. Optimal control in networks of pipes and canals. SIAM J. Control Optim. 48 (2009), 2032-2050. Zbl1196. 35135 MR2516198

11. Dafermos, C. M. Hyperbolic conservation laws in continuum physics, volume 325 of Grundlehren der Mathematischen Wissenschaften [Fundamental Principles of Mathematical Sciences]. Springer-Verlag, Berlin, second edition, 2005. Zbl1196.35001 MR2574377 
12. Fazio, R. \& LeVeque, R. J. Moving-mesh methods for one-dimensional hyperbolic problems using CLAWPACK. Comput. Math. Appl. 45 (2003), 273-298. Numerical methods in physics, chemistry, and engineering. Zbl1035.65094 MR1991370

13. Fernández, M. Á., Milišić, V. \& Quarteroni, A. Analysis of a geometrical multiscale blood flow model based on the coupling of ODEs and hyperbolic PDEs. Multiscale Model. Simul. 4 (2005), 215-236 (electronic). Zbl1085.35095 MR2164715

14. FILIPPOV, A. F. Differential equations with discontinuous righthand sides. Kluwer Academic Publishers Group, Dordrecht, 1988. Translated from the Russian. MR1028776

15. Lattanzio, C. , Maurizi, A. \& Piccoli, B. Moving bottlenecks in car traffic flow: a PDE-ODE coupled model. SIAM J. Math. Anal. 43 (2011), 50-67. Zbl1227 . 35206 MR2765683

16. LeVeque, R. J. Finite volume methods for hyperbolic problems. Cambridge Texts in Applied Mathematics. Cambridge University Press, Cambridge, 2002. Zbl1010.65040 MR1925043 